\title{
Evaluation of corneal optical properties
} in subjects wearing hydrogel etafilcon A contact lenses and the effect of administering mannitolenriched sodium hyaluronate ophthalmic solution

\author{
This article was published in the following Dove Press journal: \\ Clinical Ophthalmology \\ 21 November 2014 \\ Number of times this article has been viewed
}

\author{
Marco Lombardo 1,2 \\ Marianna Rosati' \\ Marco Pileri ${ }^{3}$ \\ Domenico Schiano- \\ Lomoriello' \\ Sebastiano Serrao' \\ 'Fondazione G.B. Bietti IRCCS, \\ ${ }^{2}$ Vision Engineering Italy Srl, ${ }^{3} \mathrm{Azienda}$ \\ Ospedaliera San Giovanni-Addolorata, \\ Rome, Italy
}

Background: The purpose of this study was to evaluate the effect of daily administration of mannitol-enriched sodium hyaluronate ophthalmic solution on the corneal optical properties of subjects wearing low Dk hydrogel (etafilcon A) contact lenses (CLs).

Methods: Forty-five subjects wearing etafilcon A CLs daily for more than 6 months were recruited into this pilot study. Fifteen of the subjects administered a $10 \%$ mannitol-enriched $0.05 \%$ sodium hyaluronate solution (study group) once daily and 30 subjects did not administer any ophthalmic solution (control group). The subjects were examined at baseline and one month after recruitment. Changes in central corneal thickness (CCT) and corneal light backscatter were evaluated by Scheimpflug imaging (Pentacam HR). Changes in corneal total high-order aberration, corneal spherical aberration, coma, and trefoil were evaluated using the OPD scan II.

Results: At one month, corneal light backscatter decreased significantly in the study group $(\leq 18.30$ arbitrary units; $P<0.05)$ and this was highly correlated with a decrease in CCT $(R=0.81$; $P=0.04)$. The decrease in corneal total high-order aberration, spherical aberration, and coma was significantly higher in the study group than in the control group $(P<0.05)$. No changes in corneal light backscatter or CCT were found in the control group during follow-up.

Conclusion: Once-daily administration of a mannitol-enriched lubricant ophthalmic solution was effective for improving the corneal optical quality and reducing corneal swelling in subjects wearing low Dk hydrogel (etafilcon A) CLs during one month follow-up.

Keywords: corneal optical quality, corneal high-order aberrations, Scheimpflug imaging, corneal backscattering, contact lens, hypertonic lubricant solution

\section{Introduction}

During the last two decades, contact lenses (CLs) have gained widespread acceptance. More than 125 million people wear CLs every day worldwide. The age of CL users is on average 31 years and about two-thirds of users are female. ${ }^{1}$

The function and optical performance of the corneal tissue after CL wear is currently receiving much attention. The structural integrity of the first optical surface of the cornea as well as normal corneal microstructure and metabolism are fundamental for the optical quality of the cornea. ${ }^{2}$ The measurement of corneal high-order wavefront aberration (WA) and corneal light backscattering ensures objective evaluation of the optical performance of corneal tissue. ${ }^{3}$ In subjects wearing CLs, corneal wavefront sensing and Scheimpflug imaging have been shown to be valuable for assessing the effect of lubricant ophthalmic solution on the optical quality of the cornea. ${ }^{4-8}$ Increased corneal high-order WA has been mainly related to surface irregularities and/or tear
Correspondence: Marco Lombardo Instituto Di Ricovero e Cura a Carattere Scientifico, Fondazione G.B. Bietti, Via Livenza 3, 00198 Roma, Italy Tel +39068535 6727

Fax +390684242333

Email mlombardo@visioeng.it 
film instability. ${ }^{9-13}$ Increased corneal light backscatter has been related to the localized corneal epithelial and stromal response to CLs, ${ }^{14,15}$ and specifically to moderate corneal swelling associated with prolonged wearing of CLs. ${ }^{16}$

Hydrogel materials still represent $25 \%$ of the global CL market. ${ }^{1}$ Prolonged wearing of these types of CL has been associated with moderate corneal swelling due to their relatively low oxygen transmissibility (Dk); specifically, etafilcon A materials have been associated with a greater risk of corneal swelling than other hydrogel CLs (eg, nelfilcon A, lotrafilcon A). ${ }^{17-19}$ In the laboratory, use of hyperosmolar ophthalmic solutions has been shown to be effective in minimizing corneal swelling without altering corneal epithelial cells. ${ }^{20,21}$ The purpose of this pilot study was to evaluate the effect of topical administration of a 10\% mannitol-enriched $0.05 \%$ sodium hyaluronate ophthalmic solution on corneal thickness and corneal optical properties in subjects wearing etafilcon A CLs during one month of follow-up.

\section{Materials and methods}

Subjects wearing hydrogel etafilcon A CLs were recruited for this study. The study followed the tenets of the Declaration of Helsinki and was approved by the local ethics committee (Azienda Sanitaria Locale Roma A, Rome, Italy). Informed consent was obtained from all subjects after full explanation of the study protocol. The inclusion criteria were age $\geq 18$ years, a manifest spherical equivalent refraction from +4.0 to $-8.0 \mathrm{D}, 10 / 20$ or better best corrected visual acuity (BCVA), and wearing of etafilcon A CLs for more than 6 months and for more than 6 hours daily. The exclusion criteria were previous eye surgery, concomitant ocular pathology, Schirmer $1<10 \mathrm{~mm}$, tear film break-up time $\leq 5$ seconds, daily topical administration of lubricant solutions or drug therapies, and chronic systemic disease.

Eligible subjects underwent a complete ocular examination, including Scheimpflug imaging by Pentacam HR (Oculus GmbH, Wetzlar, Germany) and corneal topography/ aberrometry by OPD Scan II (Nidek Co Ltd, Tokyo, Japan) both at baseline and at one month after recruitment. Examinations were performed in the afternoon ( $2 \mathrm{pm}$ to $6 \mathrm{pm}$ ), 90 minutes after discontinuing use of CLs in all cases. BCVA and topographic measurements were repeated twice with a 10-minute interval in between tests. Schirmer test 1 and break-up time tests were performed to evaluate production and stability of tear film. Slit-lamp biomicroscopy was performed to monitor for any CL-related complications according to the Efron grading scale. ${ }^{22}$ Subjects were evaluated by two expert corneal specialists (ML and SS) to minimize intersubject variance in grading of complications.

\section{Treatment}

Subjects recruited into the study group received topical administration of a $10 \%$ mannitol-enriched $0.05 \%$ sodium hyaluronate ophthalmic solution (Terso, DMG Italia Srl, Italy; $1,000 \pm 200 \mathrm{mOsmol} / \mathrm{mL}$ ). They were required to administer one drop daily in the morning before wearing their CLs. Subjects in the control group did not receive any lubricant ophthalmic solution.

\section{Analysis of corneal data}

Pentacam HR and OPD scan II measurements were repeated three times for each eye, and the best test image was chosen for analysis. Central corneal thickness (CCT) was evaluated using the Pentacam HR software. Corneal light backscattering was quantified using Scheimpflug photography. Each eye was photographed using the "3D-Scan 25 pictures" program of the Pentacam HR. Two of the Scheimpflug images, with a superior and temporal camera position, were exported as 8 bit.bmp format files (0-256 intensity gray levels) and processed using the ImageJ image-analysis program (http://imagej.nih.gov/ij, NIH, USA) both at baseline and after one month of follow-up. For each eye, the baseline and onemonth images acquired along the same corneal meridian were registered using a cross-correlation algorithm in order to determine their relative position displacements. The baseline image was used as a reference frame and a template of image features was selected on the reference frame in order to improve the algorithm precision. Using a bicubic interpolation for translation, alignment with subpixel precision at the $x$ and $y$ directions was obtained. This procedure permitted precise measurement of corneal light backscattering at exactly the same points before and after treatment. Corneal light backscattering was then measured using the "Line Tool" of ImageJ at 50 points along a line perpendicular to the corneal optical center from Scheimpflug images. The light backscattering pretreatment data were used for the baseline values and were compared with data obtained at one month. Average and peak light-scattering values were assessed for each line (Figure 1).

The optical quality of the corneal surface was evaluated by calculating the root-mean-square error of higher-order aberrations (HOA) over a simulated $6 \mathrm{~mm}$ pupil (ie, scotopic pupil condition). ${ }^{23,24}$ The corneal high-order WA was computed with respect to the line of sight, ie, the center of the entrance pupil. ${ }^{25} \mathrm{We}$ considered corneal total HOA up to sixth order spherical aberration, coma, and trefoil.

\section{Statistical analysis}

Eligible subjects were consecutively allocated to the study group or control group according to a 1:2 scheme. The statistical 


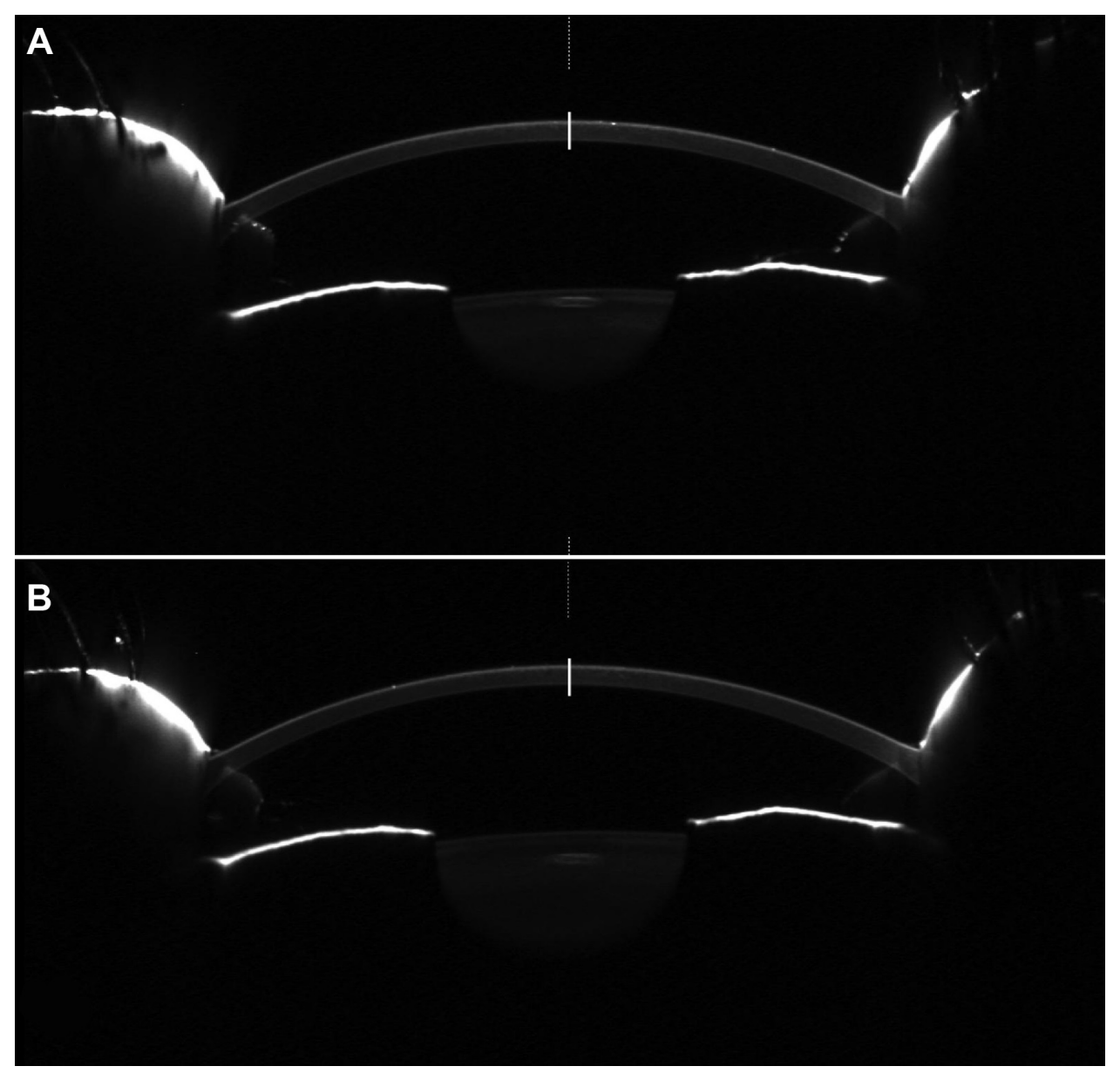

Figure I Scheimpflug photography was used to determine the changes of corneal light backscattering in all subjects.

Notes: For each subject, baseline (A) and one month (B) Scheimpflug images, acquired along the main corneal meridians, were exported and processed in ImageJ. The images were thus registered in order to measure light backscattering at exactly the same points across the corneal optical center (white lines; case AI0).

test was calculated (using GPower version 3.1.2) having 82\% power to determine differences of 10 arbitrary units (AU; SD $15 \%$ from the mean) in the corneal light backscatter and of $0.04 \mu \mathrm{m}$ (standard deviation [SD] 20\% from the mean) in the corneal high-order WA between the study and control group (total cases 45).

Analysis of corneal backscattering data and corneal HOA were performed by two authors (MP and DSL) masked to the eye group. Statistical Package for the Social Sciences version 17 software (SPSS Inc, Chicago, IL, USA) was used for all statistical testing. Two sets of BCVA and topography measurements taken at 10-minute intervals from each other did not show any difference, so only the first measurement was used for the statistical analysis. High correlations were found between the corneal WA data and backscatter profiles for the right and left eyes at baseline. Hence, only one eye for each subject was used for further calculations. One-way analysis of variance was used to compare the baseline data with the one-month data in each group. Statistical comparison of topographic, aberration, and CCT values between groups was performed using multivariate analysis of variance. The Pearson coefficient was used to estimate the correlation between changes in corneal light backscatter and CCT. Differences with a $P$-value $\leq 0.05$ were considered to be statistically significant.

\section{Results}

Forty-five subjects, comprising 30 females and 15 males, were recruited into the study. The mean subject age was $30.75 \pm 7.03$ (range: 19-40) years. Both Schirmer 1 and break-up time tests were within normal limits in all subjects. There were no serious biomicroscopic signs of complications of CL wear (Efron grading scale $<1$ ) in any case.

The characteristics of both study groups are summarized in Table 1. All subjects were wearing hydrogel ionic CLs composed of etafilcon A (HEMA/MA), with a water content of $58 \%$, a center thickness of $0.084 \mathrm{~mm}$, and an oxygen transmissibility Dk/t $=33 \times 10^{-9} \mathrm{~cm} / \mathrm{sec}\left(1\right.$-day Acuvue ${ }^{\circledR}$ Moist $^{\circledR}$, Johnson \& Johnson Vision Care, Inc., Jacksonville, FL, USA). No subject had worn any other type of CL in the past 6 months. 
Table I Subject characteristics

\begin{tabular}{llllll}
\hline & & Age (years) & Sex (M:F) & $\begin{array}{l}\text { Manifest spherical } \\
\text { equivalent refraction (D) }\end{array}$ & $\begin{array}{l}\text { Daily CL wearing } \\
\text { time (hours) }\end{array}$ \\
\hline Study group $(\mathbf{n = 1 5 )}$ & Mean \pm SD & $31.00 \pm 5.60$ & $3: 12$ & $-3.07 \pm 2.19$ & $9.14 \pm 2.32$ \\
& Range & $24-37$ & & $+1,-6$ & $6-12$ \\
Study males & Mean \pm SD & $35.5 \pm 1.73$ & - & $-3.50 \pm 1.71$ & $10.00 \pm 1.63$ \\
Study females & Mean \pm SD & $29.9 \pm 5.63$ & - & $-2.90 \pm 2.41$ & $8.80 \pm 2.53$ \\
Control group $(\mathbf{n = 3 0 )}$ & Mean \pm SD & $30.63 \pm 7.69$ & $12: 18$ & $-2.93 \pm 2.59$ & $9.13 \pm 1.94$ \\
& Range & $21-40$ & & $+3,-7$ & $6-12$ \\
Control males & Mean \pm SD & $31.82 \pm 8.34$ & - & $-3.18 \pm 1.47$ & $9.45 \pm 2.21$ \\
Control females & Mean \pm SD & $29.9 \pm 7.44$ & - & $-2.75 \pm 3.17$ & $8.95 \pm 1.81$ \\
\hline
\end{tabular}

Abbreviations: $\mathrm{CL}$, contact lens; $\mathrm{SD}$, standard deviation.

There were no statistically significant differences ( $P>0.05$, analysis of variance) between the refractive errors and corneal parameters in the study group and the control group at baseline. Subjects did not complain of any intolerance to daily administration of the hypertonic lubricant solution. No side effects were recorded in this series. At baseline, BCVA was better than 20/25 in all subjects and was stable during one month of follow-up.

The changes in corneal light backscattering did not differ between corresponding horizontal and vertical measurement points during follow-up; therefore, the mean of the corresponding points was used in all cases. The changes of corneal light backscattering values were significantly different $(P<0.05)$ between the study group and the control group during follow-up. At one month, we found a decrease in the peak and average intensity of corneal light backscattering with respect to baseline in the study group (Figure 2). The average peak intensity decreased from $93.83 \pm 11.45 \mathrm{AU}$ at baseline to $75.50 \pm 13.3 \mathrm{AU}$ at one month $(<19.3 \%$, ranging between $6.3 \%$ and $36.0 \%$ across subjects; $P<0.05$ ). The average light backscattering decreased from $30.76 \pm 5.90$ AU to $23.64 \pm 2.48$ AU during follow-up $(<23.2 \%$, ranging between $15.1 \%$ and $38.1 \%$ across subjects; $P<0.05)$. This decrease was significantly correlated with corneal thinning $(R=0.81 ; P=0.04)$, as shown in Figure 3. At one month, no significant change in corneal light backscatter was found in the control group (peak $90 \pm 9.77 \mathrm{AU}$ and mean $28.17 \pm 2.12 \mathrm{AU}$ ) compared with baseline (peak $88 \pm 9.48 \mathrm{AU}$ and mean $27.85 \pm 2.25 \mathrm{AU}$ ).

Table 2 summarizes the average baseline and one-month corneal high-order WA data and CCT values in both study groups. The changes in corneal total HOA $(P=0.04)$, spherical aberration $(P=0.05)$, trefoil $(P=0.04)$, and coma $(P=0.03)$

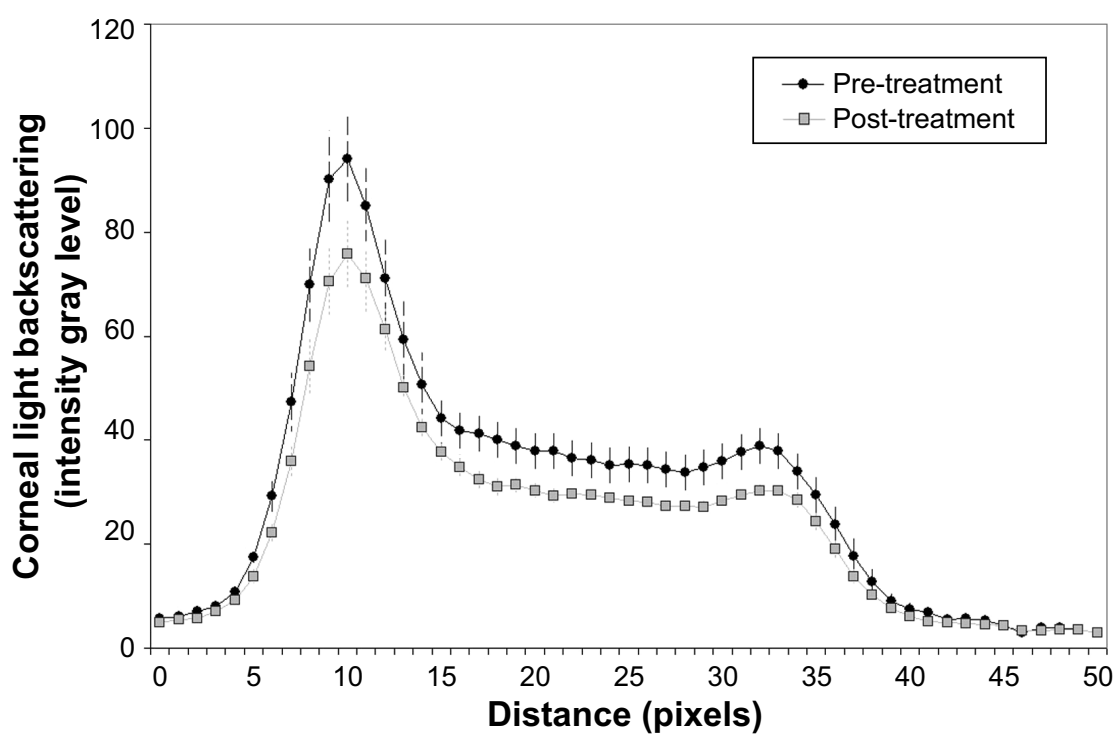

Figure 2 Average \pm standard deviation corneal light backscattered distribution at baseline (black line) and one month after daily administration of hypertonic lubricant solution (gray line).

Note: The topical administration of the hypertonic, hyaluronate-enriched, ophthalmic solution was effective to decrease corneal light backscatter in the study group during one month of follow-up. 


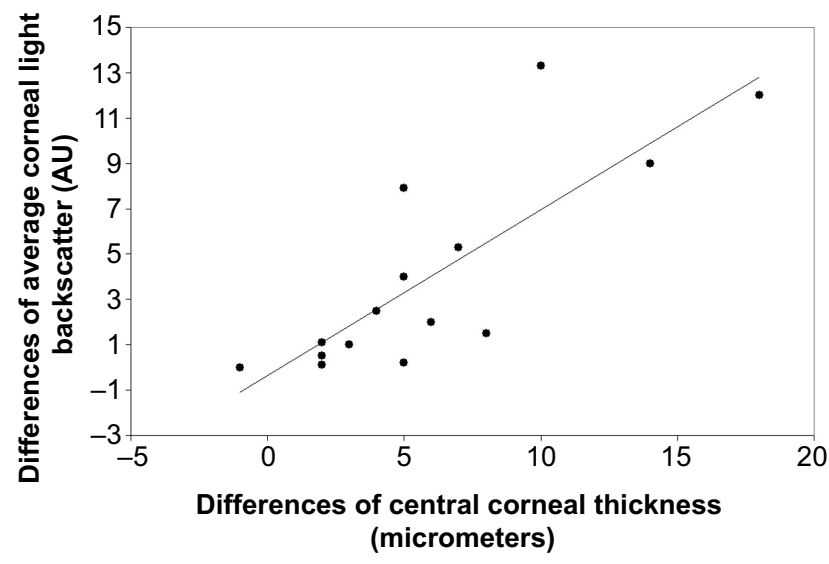

Figure 3 Correlation scattergram between the changes induced by the hypertonic, hyaluronate-enriched, topical treatment in the central corneal thickness and the average corneal light backscatter in each subject of the study group.

Note: The decrease in corneal light backscatter was statistically significant and highly correlated with corneal deswelling $(R=0.81 ; P=0.04)$.

were significantly different between the study group and the control group during follow-up. Corneal total HOA and spherical aberration decreased on average by $9 \%$ with respect to baseline, and coma and trefoil decreased on average by $15 \%$ in the study group ( $P>0.05$; Figure 4$)$. Corneal total HOA and spherical aberration increased $(\geq 9 \% ; P>0.05)$ in the control group, while coma and trefoil values were essentially stable during follow-up (changes $\leq 4 \% ; P>0.05$ ).

CCT decreased in the study group $(-6 \mu \mathrm{m},<1.1 \%$; $P=0.04)$ but was stable in the control group $(0 \mu \mathrm{m}, P>0.05)$ during follow-up. Figure 5 shows the corneal maps in a representative case from the study group at baseline and after one month of follow-up.

\section{Discussion}

Forty-five subjects wearing hydrogel etafilcon A CLs were recruited for this pilot study. Of these subjects, 15 were allocated to the study group and administered a $10 \%$ mannitol-enriched $0.05 \%$ sodium hyaluronate ophthalmic solution once daily 20 minutes before wearing their CLs, with the remaining 30 subjects being followed as a control group. The study design had enough statistical power $(>80 \%)$ to demonstrate the efficacy of daily administration of a hypertonic ophthalmic lubricant solution on corneal optical properties in subjects wearing etafilcon A CLs. The corneal optical properties were investigated by corneal aberrometry and Scheimpflug photography. Baseline and onemonth follow-up examinations were done 90 minutes after removal of the CLs. This procedure was based on previous work $^{16}$ showing that both CCT and the profile of corneal backscattered light are higher than expected immediately after CL removal, then decrease during the subsequent hour. This procedure allowed us to remove an important bias from our study, in that we were able to quantify the actual corneal properties reliably in each case. In addition, measurements were taken in the afternoon because this is the time of the day when the eye is most physiologically stable. ${ }^{26}$ Indeed, corneal measurements were acquired twice with a 10-minute interval in between, and the measurements did not show any differences, thus confirming the results reported by Koh et al. ${ }^{4}$

Variation in corneal surface smoothness and anterior transparency can affect corneal light backscatter, which can be estimated by different approaches, such as optical coherence tomography, Scheimpflug photography, and psychophysical methods (glare testing, adaptive optics visual simulator with diffuser, direct compensation method). ${ }^{16,27-29} \mathrm{We}$ found a significant decrease in both peak and average corneal light backscattering by Scheimpflug imaging in the study group. The decrease in corneal light backscatter was highly correlated with corneal deswelling. No changes in corneal light backscatter or CCT were found in the control group during follow-up, thus confirming the valuable effect of the hypertonic topical treatment on corneal hydration in patients wearing etafilcon A CLs. This type of CL was previously associated with increased corneal swelling (on average by 5.5\%) after 8 hours of closed-eye wear (overnight) with respect to silicone hydrogel lenses (on average $1.5 \%$ swelling). ${ }^{30,31}$ Lambert and

Table 2 Average \pm standard deviation corneal high-order wavefront aberration and CCT values and CCT values at baseline and at one month follow-up

\begin{tabular}{|c|c|c|c|c|c|}
\hline & Total HOA $(\mu \mathrm{m})$ & SA $(\mu \mathrm{m})$ & Coma $(\mu \mathrm{m})$ & Trefoil $(\mu \mathrm{m})$ & ССТ $(\mu \mathrm{m})$ \\
\hline \multicolumn{6}{|c|}{ Study group $(n=15)$} \\
\hline Baseline & $0.38 \pm 0.08$ & $0.26 \pm 0.05$ & $0.20 \pm 0.09$ & $0.14 \pm 0.06$ & $561 \pm 24$ \\
\hline One month & $0.35 \pm 0.08 *$ & $0.24 \pm 0.04 *$ & $0.17 \pm 0.09 *$ & $0.12 \pm 0.09 *$ & $555 \pm 18^{*}$ \\
\hline \multicolumn{6}{|c|}{ Control group $(n=30)$} \\
\hline Baseline & $0.38 \pm 0.09$ & $0.25 \pm 0.07$ & $0.20 \pm 0.11$ & $0.15 \pm 0.07$ & $558 \pm 20$ \\
\hline One month & $0.40 \pm 0.12 *$ & $0.27 \pm 0.05^{*}$ & $0.20 \pm 0.12 *$ & $0.16 \pm 0.07 *$ & $558 \pm 21$ \\
\hline
\end{tabular}

Note: $* P<0.05$, changes between the study and control group.

Abbreviations: CCT, central corneal thickness; HOA, high-order aberration; SA, spherical aberration. 


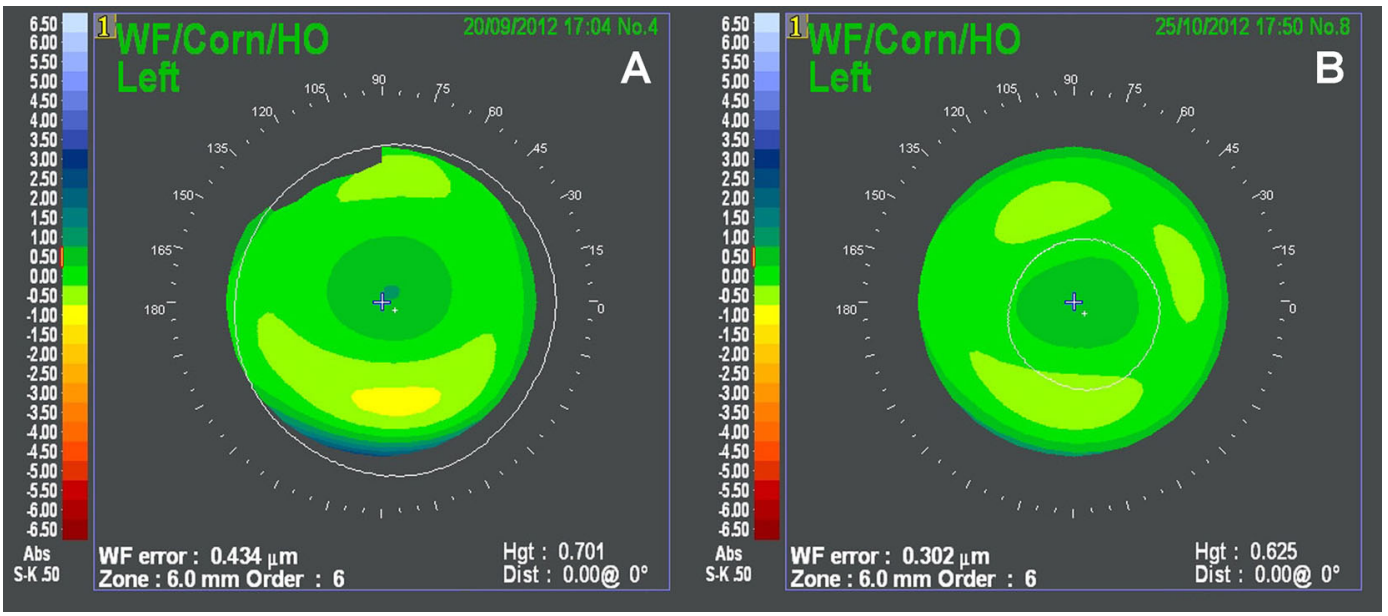

Figure 4 Baseline (A) and one month follow-up (B) corneal high-order WA over a $6.00 \mathrm{~mm}$ pupil in a representative case (A04) of the study group. One month after hypertonic treatment, corneal total HOA decreased by one third (from 0.434 to $0.302 \mu \mathrm{m}$ ) compared to the baseline visit.

Abbreviations: WA, wavefront aberration; HOA, high-order aberrations.

Klyce $^{32}$ found increased light-scattering in the epithelium during corneal swelling induced by oxygen deprivation. Farris et $\mathrm{a}^{14}$ and Korb and Exford ${ }^{33}$ reported that this phenomenon was due to a localized epithelial response to CL wear, resulting in a focal increase of backscattered light. In our study, the corneal backscattered light in the epithelium (measured as peak intensity in this study) could be related to the distortion of the surface microstructure, ${ }^{34}$ and the backscattered light in the stroma (measured as average intensity) could be related to local hydration. ${ }^{15,35}$
One month after topical administration of 10\% mannitolenriched $0.05 \%$ sodium hyaluronate solution, corneal highorder WA tended to decrease in the study group. The corneal total HOA tended to increase in the control group during follow-up, thus confirming the potential benefit of the hypertonic hyaluronate-enriched lubricant solution in minimizing irregularities of the first optical surface of the cornea. Indeed, the differences between groups were statistically significant at one month of follow-up. Corneal wavefront sensing provides objective assessment of corneal optical quality. It has

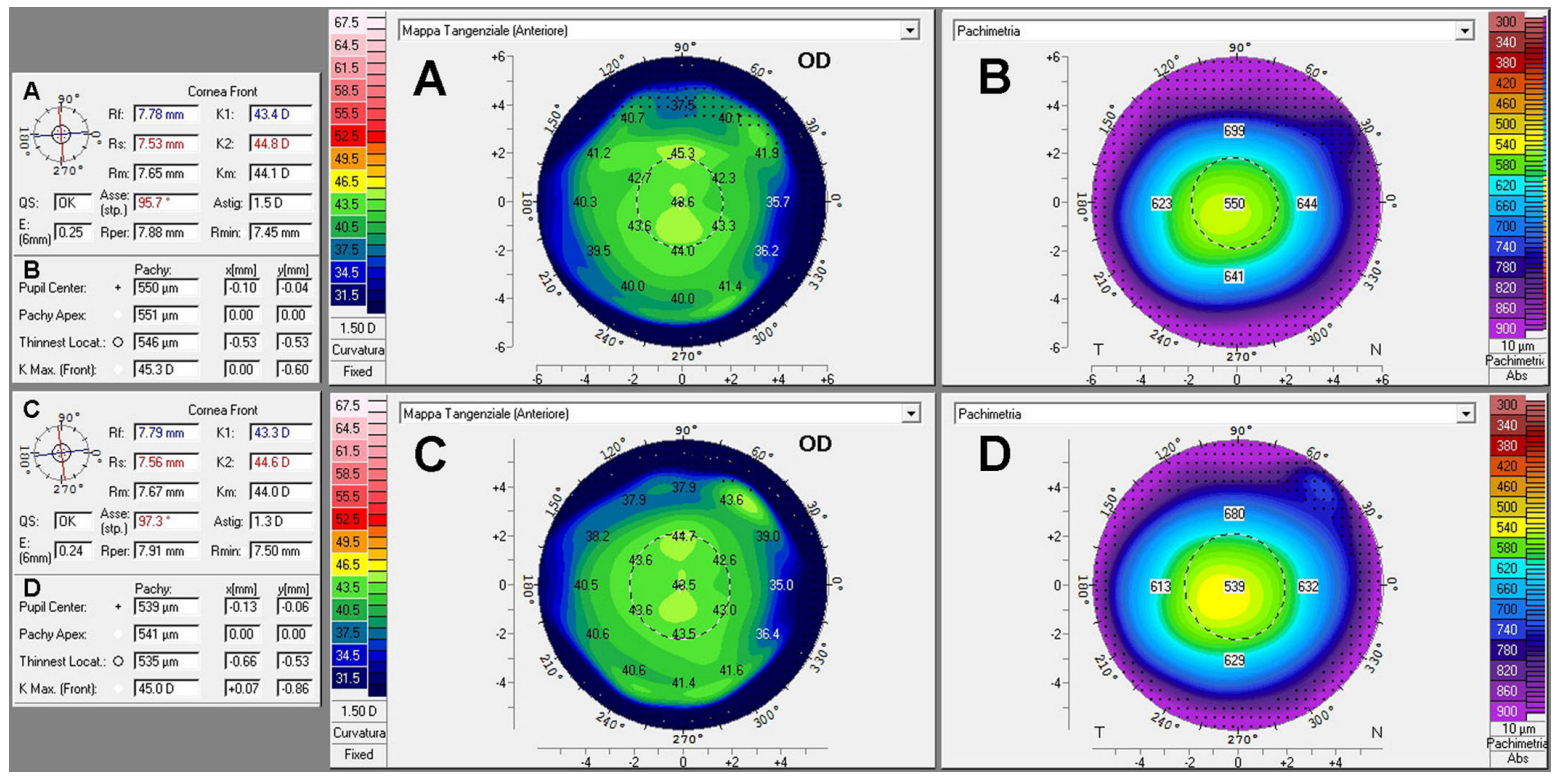

Figure 5 Baseline (A, B) and one-month follow-up (C, D) Scheimpflug corneal maps (anterior tangential and thickness maps, respectively) for a representative case (A02) in the study group. One month after hypertonic treatment, central corneal thickness decreased by II $\mu \mathrm{m}$. 
been already used for testing the effect on image quality of CL wearing as well as the benefit of lubricant ophthalmic solutions. ${ }^{3-7,36}$ Several factors can affect the smoothness of the corneal surface following wearing of CLs, and include tear film instability and corneal swelling. ${ }^{6,15,37}$ The improved optical quality of the cornea in subjects administering daily hypertonic hyaluronate-enriched lubricant solution could be ascribed to both corneal deswelling and decreased microirregularities on the corneal first surface as a result of tear film stabilization. ${ }^{10,11,15,38,39}$ No correlation was found between BCVA and decreased corneal scattering $(R=-0.08 ; P=0.87)$ or decreased total HOA $(R=-0.06 ; P=0.89)$ during follow-up.

There was no intentional sex sampling bias in our study population; however, 30 of the 45 subjects in this study were female. Nevertheless, this did not impact our results since the groups were age-matched, and had matched manifest spherical equivalent refraction values and daily CL wearing time. Additionally, in the range of age of subjects in this study, sex has not been associated with corneal aberrations and has been shown to have little or no impact on tear volume or its protein content. ${ }^{5,40,41}$ All subjects recruited into the present study had normal tear volume and film stability.

The results of this pilot study show that daily administration of a hypertonic ophthalmic solution was effective in minimizing corneal swelling due to wearing of low Dk etafilcon A CLs, while improving the optical properties of the corneal surface. None of the subjects recruited into the present study complained of side effects during follow-up.

\section{Conclusion}

In this study, we demonstrated recovery of corneal light backscattering and improvement in corneal optical quality associated with decreased CCT in subjects wearing hydrogel etafilcon CLs and applying one drop of hypertonic ophthalmic lubricant solution daily. We did not record any intolerance or side effects during one month of follow-up. A longer study is needed to assess the safety and efficacy of this approach and to determine whether this treatment may lead to improved visual performance (eg, contrast sensitivity) during wearing of low Dk CLs. ${ }^{6}$

\section{Acknowledgment}

The study was funded by the Italian Ministry of Health and by Fondazione Roma. The authors are grateful to DMG Italia Srl for providing the hypertonic ophthalmic solution used in this study.

\section{Disclosure}

The authors report no conflicts of interest in this work.

\section{References}

1. Nichols JJ. Contact lenses annual report: 2012. Contact Lens Spectrum. 2013;28:24-29.

2. Leung BK, Bonanno JA, Radke CJ. Oxygen-deficient metabolism and corneal edema. Prog Ret Eye Res. 2011;30:471-492.

3. Lombardo M, Lombardo G. New methods and techniques for sensing the wave aberration of human eyes. Clin Exp Optom. 2009;92:176-186.

4. Koh S, Maeda N, Hamano T, et al. Effect of internal lubricating agents of disposable soft contact lenses on higher-order aberrations after blinking. Eye Contact Lens. 2008;34:100-105.

5. Keir N, Simpson T, Fonn D. Visual and optical performance of silicone hydrogel contact lenses for moderate myopia. J Optom. 2010;3: 149-157.

6. Pons AM, Lorente A, Albarrán C, Montés R, Artigas JM. Characterization of the visual performance with soft daily wear disposable contact lenses. Ophthalmic Physiol Opt. 1998;18:40-48.

7. Stahl U, Willicox M, Stapleton F. Role of hypo-osmotic saline drops in ocular comfort during contact lens wear. Cont Lens Anterior Eye. 2010; 33:68-75.

8. Lira M, Oliveira ME, Franco S. Comparison of the tear film clinical parameters at two different times of the day. Clin Exp Optom. 2011; 94:557-562.

9. Shen J, Thibos LN. Peripheral aberrations and image quality for contact lens correction. Optom Vis Sci. 2011;88:1196-1205.

10. LuF, Mao X, Qu J, Xu D, He JC. Monochromatic wavefront aberrations in the human eye with contact lenses. Optom Vis Sci. 2003;80:135-141.

11. Montés-Micó R, Cerviño A, Ferrer-Blasco T, García-Lázaro S, MadridCosta D. The tear film and the optical quality of the eye. Ocul Surf. 2010;8:185-192.

12. Stahl U, Willicox M, Stapleton F. Osmolarity and tear film dynamics. Clin Exp Optom. 2012;95:3-11.

13. Szczesna-Iskander DH, Iskander DR, Read SA, Alonso-Caneiro D. Noninvasive in vivo assessment of soft contact lens type on tear film surface quality. Invest Ophthalmol Vis Sci. 2012;53:525-531.

14. Farris RL, Kubota Z, Mishima S. Epithelial decompensation with corneal contact lens wear. Arch Ophthalmol. 1971;85:651-660.

15. Wang J, Fonn D, Simpson TL, Jones L. The measurement of corneal epithelial thickness in response to hypoxia using optical coherence tomography. Am J Ophthalmol. 2002;133:315-319.

16. Wang J, Simpson TL, Fonn D. Objective measurements of corneal lightbackscatter during corneal swelling by optical coherence tomography. Invest Ophthalmol Vis Sci. 2004;45:3493-3498.

17. Martin R, Izquierdo M, Saber A. Investigation of posterior corneal curvature in CL-induced corneal swelling. Cont Lens Anterior Eye. 2009;32:288-293.

18. Martin R, Nuñez L, Sastre J, de Juan V, Rodriguez G. Constancy of the Orbscan acoustic factor to detect contact lens-induced corneal swelling. Clin Exp Optom. 2011;94:352-360.

19. Martin R, de Juan V, Rodriguez G, Fonseca S, Martin S. Contact lensinduced corneal peripheral swelling differences with extended wear. Cornea. 2008;27:976-979.

20. Schrage N, Wuestemeyer H, Langefeld S. Do different osmolar solutions change the epithelial surface of the healthy rabbit cornea? Graefes Arch Clin Exp Ophthalmol. 2004;242:668-673.

21. Insler MS, Benefield DW, Ross EV. Topical hyperosmolar solutions in the reduction of corneal edema. CLAO J. 1987;13:149-151.

22. Efron N. Grading scales for contact lens complications. Ophthalmic Physiol Opt. 1998;18:182-186.

23. Serrao S, Lombardo G, Lombardo M, Palombi M, Roberts CJ. Corneal topography six years after photorefractive keratectomy for myopia and myopic astigmatism. J Refract Surg. 2009;25:451-458. 
24. Serrao S, Lombardo G, Ducoli P, Lombardo M. Long-term corneal wavefront aberration variations after photorefractive keratectomy for myopia and myopic astigmatism. J Cataract Refract Surg. 2011; 37:1655-1666.

25. Lombardo M, Lombardo G. Wave aberration of human eyes and new descriptors of image optical quality and visual performance. J Cataract Refract Surg. 2010;36:313-331.

26. Martin R, de Juan V, Rodriguez G, Cuadrado R, Fernandez I. Measurement of corneal swelling variations without removal of the contact lens during extended wear. Invest Ophthalmol Vis Sci. 2007;48: 3043-3050.

27. Beckman Rehnman J, Janbaz CC, Behndig A, Linden C. Spatial distribution of corneal light scattering after corneal collagen crosslinking. J Cataract Refract Surg. 2011;37:1939-1944.

28. Binder PS, Bosem M, Weinreb RN. Scheimpflug anterior segment photography assessment of wound healing after myopic excimer laser photorefractive keratectomy. J Cataract Refract Surg. 1996;22:205-212.

29. Møller-Pedersen T, Cavanagh HD, Petroll WM, Jester JV. Corneal haze development after PRK is regulated by volume of stromal tissue removal. Cornea. 1998;17:627-639.

30. Steffen RB, Schnider CM. The impact of silicone hydrogel materials on overnight corneal swelling. Eye Contact Lens. 2007;33:115-120.

31. Hamano H, Maeda N, Hamano T, Mitsunaga S, Kotani S. Corneal thickness change induced by dozing while wearing hydrogel and silicone hydrogel lenses. Eye Contact Lens. 2008;34:56-60.

32. Lambert S, Klyce S. The origins of Sattler's veil. Am JOphthalmol. 1981; 91:51-56
33. Korb DR, Exford JM. The phenomenon of central circular clouding. J Am Optom Assoc. 1968;39:223-230.

34. Rozema JJ, Trau R, Verbruggen KH, Tassignon MJ. Backscattered light from the cornea before and after laser-assisted subepithelial keratectomy for myopia. J Cataract Refract Surg. 2011;37:1648-1654.

35. Silverman RH, Patel MS, Gal O, et al. Effect of corneal hydration on ultrasound velocity and backscatter. Ultrasound Med Biol. 2009;35: 839-846.

36. Szczesna DH, Jaroński J, Kasprzak HT, Stenevi U. Interferometric measurements of dynamic changes of tear film. J Biomed Opt. 2006;11: 34028.

37. Rho CR, Pandey C, Kim SY, Kim MS. Corneal swelling caused by conventional and new-design low-Dk soft contact lenses following a 10-day daily wear trial regime. Cont Lens Anterior Eye. 2014;37:38-43.

38. Airiani S, Rozell J, Lee SM, Braunstein RE. The effect of lubricant eye drops on ocular wavefront aberrations. J Refract Surg. 2005;21: 709-715.

39. Hirai S, Kawahara M, Sakamoto K, Kimura A, Nakamura M. Effects of various lubricants on corneal surface regularity in rabbits. Ocul J Pharmacol Ther. 2005;21:376-381.

40. Goto T, Klyce SD, Zheng X, Maeda N, Kuroda T, Ide C. Gender and agerelated differences in corneal topography. Cornea. 2001;20:270-276.

41. Sullivan DA, Hann LE, Yee L, Allansmith MR. Age- and gender-related influence on the lacrimal gland and tears. Acta Ophthalmol (Copenh). 1990;68:188-194.
Clinical Ophthalmology

\section{Publish your work in this journal}

Clinical Ophthalmology is an international, peer-reviewed journal covering all subspecialties within ophthalmology. Key topics include: Optometry; Visual science; Pharmacology and drug therapy in eye diseases; Basic Sciences; Primary and Secondary eye care; Patient Safety and Quality of Care Improvements. This journal is indexed on

\section{Dovepress}

PubMed Central and CAS, and is the official journal of The Society of Clinical Ophthalmology (SCO). The manuscript management system is completely online and includes a very quick and fair peer-review system, which is all easy to use. Visit http://www.dovepress.com/ testimonials.php to read real quotes from published authors. 